grün. Ganze übrige Unterseite hell olivengrün, in der Mittellinie etwa $1 \mathrm{~cm}$ breit gelb. - Schwanzfedern unterseits olivengrün mit schmalen gelblichen Rändern, oberseits, von der Rïckenfärbung deutlich verschieden, olivenbraun mit schmalen grünlichen Rändern. - Es liegt nur ein weibliches Exemplar vor, das folgende Maasse aufweist:

Long. tot. $23 \mathrm{~cm}$. Ala $10,5 \mathrm{~cm}$. Cauda $9,6 \mathrm{~cm}$. Culmen $2,5 \mathrm{~cm}$. Tarsus $2,0 \mathrm{~cm}$.

Typ.: Collection Nehrkorn.

Ich benenne diese Art zu Ehren des eifrigen Förderers der Naturwissenschaften, besonders der Botanik, des Cardinals, Erzbischofs von Kolocsa in Ungarn, Herrn Dr. Ludwig Haynald.

Braunschweig, Herzogl. Naturhistorisches Museum,

8. Mai 1890.

\title{
Die von Herrn Dr. Platen und dessen Gemahlin im Sommer 1889 bei Davao auf Mindanao gesammelten Vögel,
}

welche ich durch Herrn Oberamtmann A. Nehrkorn in Riddagshausen kürzlich zur wissenschaftlichen Bestimmung und Bearbeitung erhielt, habe ich in der Sitzung des Vereins für Naturwissenschaft zu Braunschweig vom 13. März 1890 einer ersten wissenschaftlichen Besprechung unterzogen; der Bericht darüber ist in den Braunschweigischen Anzeigen vom 27. März 1890 Nr. 73 und vom 15. April 1890 Nr. 87 erschienen. In der letzteren Nummer besonders wurden zuerst die Beschreibungen von 7 neuen Arten veröffentlicht, welche ich am Schlusse dieser Zusammenstellung unter Hinzufügung einiger näheren Angaben über den Verbleib des typischen Exemplare und einiger Maasse wörtlich wiederhole. Den wesentlichsten Inhalt meiner damaligen Besprechung beehre ich mich, im Folgenden der Versammlung der Allgemeinen Deutschen Ornithologen-Gesellschaft gegenüber zu wiederholen.

Nach den Platen'schen Sammlungen sind zum ersten Ma.e für Mindanao nachgewiesen die folgenden Arten:

Falconidae.

*1. Lophospiza trivirgata (Temm.).

*2. Spizaëtus limnaëtus (Horsf.).

*3. Baza magnirostris G. R. Gray.

*4. Circus spilonotus Kaup. 
Die von Dr. Platen auf Mindanao gesammelten Vögel.

Strigidae.

*5. Ninox macroptera W. Blas.

Cuculidae.

*6. Cuculus canoroides S. Müll.

Alcedidae.

*7. Ceyx Platenae sp. nov.

$\odot 8$. Ceyx Margarethae sp. nov.

Laniidae.

*9. Lanius lucionensis Linn.

*10. Hyloterpe philippinensis Walden.

$$
\text { Muscicapidae. }
$$

*11. Rhinomyias ruficauda (Sharpe).

$\odot$ 12. Zeocephus cinnamomeus Sharpe.

*13. Muscicapula mindanensis sp. nov.

Hirundinidae.

*14. Hirundo javanica Sparrm.

$$
\text { Oriolidae. }
$$

*15. Oriolus Steerii Sharpe.

$$
\text { Brachypodidae. }
$$

*16. Phyllornis Alavipennis Tweeddale.

$\odot$ 17. Micropus Nehrkorni sp. nov.

$$
\text { Timeliidae. }
$$

*18. Megalurus ruficeps Tweeddale.

$\odot$ 19. Mixornis capitalis Tweeddale.

*20. Mixornis Plateni sp. nov.

*21. Ptilopyga mindanensis sp. nov.

Dicaeidae.

○22. Prionochilus olivaceus Tweeddale.

Nectariniidae.

* 23. Arachnothera Alammifera Tweeddale.

*24. Arachnothera Clarae sp. nov.

Eurylaimidae.

*25. Sarcophanops Steerii Sharpe.

$$
\text { Columbidae. }
$$

*26. Janthoenas griseigularis Walden.

Scolopacidae.

*27. Numenius variegatus (Scop.).

*28. Totanus glottis (Linn.)

Rallidae.

*29. Amaurornis olivaceus Meyen. 
Ardeidae.
*30. Nycticorax manillensis Vigors.
Anatidae.

$\odot 31$. Dendrocygna guttata Forsten.

Von den mit einem Kreise $\odot$ bezeichneten Arten befinden sich die Belegs- bezw. typischen Exemplare nur in der Sammlung des Herrn Oberamtmann Nehrkorn in Riddagshausen, von den mit einem Stern * bezeichneten dagegen a u ch, z. Th. allein, im Herzoglichen Naturhistorischen Museum. Von der rothbäuchigen Glanzdrossel: Erythropitta, sind nebeneinander Exemplare gesammelt, welche als erythrogastra (Temm.) und als propinqua (Sharpe) angesprochen werden müssen, und es finden sich Uebergänge zwischen den beiden Formen, wodurch die Zusammengehörigkeit derselben zu einer einzigen Art höchst wahrscheinlich gemacht wird. - Die in den letzten Tagen (Le Naturaliste vom 1. März 1890 Nr. 72, S. 62/63) von E. O ustalet in Paris beschriebene Eisvogelart Halcyon Alfredi ist durch zwei Exemplare männlichen Geschlechts vertreten, während ein anderes vielleicht weibliches oder junges Exemplar einen Uebergang zu Halcyon Winchelli Sharpe zeigt. Es wird dadurch höcht wahrscheinlich, dass $H$. Alfredi nur auf männliche Individuen der anderen genannten Form begründet ist. Je zwei die erwähnten Gegensätze dieser Formen veranschaulichende Exemplare von Erythropitta erythrogastra (Temm.) und Halcyon Winchelli Sharpe sind dem Herzoglichen Naturhistorischen Museum in Braunschweig einverleibt.

Die in den Braunschweigischen Anzeigen vom 15. April 1889 Nr. 87 neu beschriebenen sieben Arten sind die folgenden:

1. Ptilopyga mindanensis, sehr ähnlich $P$. leucogrammica von Borneo; doch sind Kinn und Kehle rein weiss, von einem schwarzen Bartstreifen jederseits eingefasst. Die Federn der Kopfseiten, wie diejenigen des Oberkopfes und Nackens rothbraun mit schwärzlichen Rändern und hellbräunlichen Schaftstrichen. Von der Stirn zieht sich bis zum Nacken ein weisser Augenstreif. Die stark verlängerten und gelösten Rückenfedern bedecken etwa die Hälfte des Schwanzes und besitzen lange weissliche lanzettförmige Schaftflecken, welche bei natürlicher Lage der Federn grösstentheils unsichtbar sind.

Maasse: đ̛ Long. tot. $16 \mathrm{~cm}$. Ala 7,5 cm. Cauda $5,25 \mathrm{~cm}$. Culmen 1,95 cm. Tarsus $2,6 \mathrm{~cm}$. 
Maasse: + Long. tot. $16 \mathrm{~cm}$. Ala $6,9-7,2 \mathrm{~cm}$. Cauda $4,2-4,4 \mathrm{~cm}$. Culmen $1,9 \mathrm{~cm}$. Tarsus $2,55-2,7 \mathrm{~cm}$.

Typ.: Museum Brunsvicense und Colleetion Nehrkorn.

2. Mixornis Plateni, bedeutend kleiner als M. capitalis, mit welcher Form die Art, besonders von der Oberseite gesehen, eine gewisse Aehnlichkeit in der Färbung besitzt. Oberseite olivenbraun, Bürzel und Schwanzdeckfedern mehr rostfarben, am Vorderrücken weisse Schaftstriche. Ganzer Kopf, oben und an den Seiten, Nacken und Brust rothbraun mit feinen weissen Schaftstrichen. Kinn und Kehle mehr schwärzlich mit breiteren weissen Schaftflecken. Schwanzfedern einfarbig braun, an der Aussenfahne olivenfarben verwaschen. Ebenso die Schwungfedern oberseits und die oberen Flügeldeckfedern. Untere Flügeldeckfedern und Achselfedern weisslich.

Maasse: $q$ Long. tot. $12 \mathrm{~cm}$. Ala $5,5 \mathrm{~cm}$. Cauda $4,7 \mathrm{~cm}$. Culmen $1,0 \mathrm{~cm}$. Tarsus $1,5 \mathrm{~cm}$.

Typ.: Museum Brunsvicense.

3. Micropus Nehrkorni, in der Grösse etwa M. melanocephalus entsprechend, in der Färbung an $M$. melanoleucus erinnernd. Ganz schwarz oder schwarzbraun. Stirn und Zügel weisslich. Die Nacken- und seitlichen Halsfedern mit weissem, durchscheinenden Grundtheil (Andeutung eines Nackenbandes). Obere Flügeldeckfedern dunkel, nicht weiss. Durch die weisse Färbung des Grundtheiles der Aussenfahne, der 8 innersten Handschwingen und der 7 äusseren Mittelschwingen werden zwei weisse Flügelspiegel gebildet, von denen der obere durch die dunklen Flügeldeckfedern verdeckt wird. Die Achselfedern sind rein weiss mit grauem Grundtheil. - Vielleicht ist dies die bis dahin nicht wieder aufgefundene Vogelart, welche Gmelin 1788 als "Muscicapa luzoniensis“" beschrieben, aber nicht genügend kenntlich gemacht hat.

Maasse: o Long. tot. $14 \mathrm{~cm}$. Ala $7,85 \mathrm{~cm}$. Cauda $5,3 \mathrm{~cm}$. Culmen $1,25 \mathrm{~cm}$. Tarsus $1,5 \mathrm{~cm}$.

Typ.: Collection Nehrkorn.

4. Muscicapula mindanensis, etwas grösser als $M$. superciliaris (Jerd.). Stirn, Oberkopf, Kopfseiten und Rücken bläulich grau; Schwungfedern bei brauner Grundfarbe auf der Aussenfahne ähnlich verwaschen. Hinter den Augen beginnt jederseits ein breiter weisser Streifen, welcher sich bis in den Nacken zieht. Die weissen Spitzen der weichen verlängerten Federn des Hinterrückens bilden eine weisse Querbinde. Obere Schwanzdeckfedern und Schwanz- 
federn schwärzlich braun; letztere auf etwa $1 \frac{1}{2}$ Centimeter an der Basis gelblich-weiss. Ganze Unterseite vom Kinn bis zu den cnteren Schwanzdeckfedern weisslich mit undeutlichem grauen Brustband. - Vielleicht ist dies Gmelins 1788 beschriebene und seitdem nicht wieder beobachtete „Muscicapa philippensis,“ die allerdings die bedeutendere Grösse einer Nachtigall gehabt haben soll.

Maasse: $q$ Long. tot. $13 \mathrm{~cm}$. Ala $6,95 \mathrm{~cm}$. Cauda $4,5 \mathrm{~cm}$. Culmen 1,2 cm. Tarsus 2,15 cm.

Typ.: Museum Brunsvicense.

5. Arachnothera Clarae, in der Bildung des Gefieders, in der Grösse, Färbung und Schnabelform sehr ähnlich $A$. chrysogenys von Malakka, Borneo etc. Die nackte Stelle an den Wangen dehnt sich jedoch weiter nach hinten, bis hinter die Ohren, aus; die gelben Augenkreise und Bartfedern fehlen; die Schwung- und Schwanzfedern sind auf der Aussenfahne mehr goldgelb gefärbt Kinn, Kehle, Brust und Vorderleib grau, grünlich überflogen; diese Färbung geht allmählich nach dem Hinterleibe zu in ein mattes Gelbgrün über.

Maasse: đิ Long. tot. $17 \mathrm{~cm}$. Ala $9,15 \mathrm{~cm}$. Cauda $4,5 \mathrm{~cm}$. Culmen $3,75 \mathrm{~cm}$. Tarsus $1,9 \mathrm{~cm}$.

Maasse : $q$ Long. tot. $17 \mathrm{~cm}$. Ala $8,55 \mathrm{~cm}$. Cauda $3,9 \mathrm{~cm}$. Culmen $3,9 \mathrm{~cm}$. Tarsus $2,0 \mathrm{~cm}$.

Typ.: Museum Brunsvicense und Collection Nehrkorn.

6. Ceyx Platenae gehört zu den rothschnäbeligen und rothrückigen Arten dieser Gattung und scheint am nächsten verwandt C. melanura von den Philippinen zu sein. Hinterrücken bis zum Schwanz braunroth; Kopf, Nacken und Vorderrücken ebenso mit lilafarbenen Spitzenflecken. Kinn und Kehle rein weiss. Ganze Unterseite roströthlich, die Brust am dunkelsten und ganz mit Purpurviolett verwaschen. Leib heller, in der Mittellinie gelblichweiss. Schwanzfedern braunroth, die äusseren etwas dunkel verwaschen. Schulterfedern und obere Flügeldeckfedern schwärzlich mit rothbraunen Spitzen, ohne Blaufärbung. Die Flecken an den Seiten des Halses weisslich und nicht blau. Schon Sharpe scheint ein Exemplar dieser Art von Mindanao 1877 in Händen gehabt und nur mit einigem Zweifel zu melanura gezogen zu haben.

Maasse: Long. tot. $13 \mathrm{~cm}$. Ala $6,05 \mathrm{~cm}$. Cauda $2,1 \mathrm{~cm}$. Culmen $3,8 \mathrm{~cm}$. Tarsus $0,95 \mathrm{~cm}$.

Typ.: Museum Brunsvicense.

7. Ceyx Margarethae gehört $\mathrm{zu}$ den rothschnäbeligen und blau- 
rückigen Arten und ist von den verwandten Formen: C. lepida, uropygialis etc. durch eine einfarbig dunkel ultramarinblaue Oberseite (Kopf mit Einschluss der Zügel- und Ohrgegend, Nacken, Rücken, Schulterfedern und obere Schwanzdeckfedern) unterschieden. Mit derselben Farbe sind die schwärzlichen oberen Flügeldecken an den Spitzen gefärbt und die schwarzen Schwanzfedern sowie die gleichgefärbten Mittel- und inneren Handschwingen an der Aussenfahne verwaschen. Die ziemlich grossen Stirn- und Bartflecken jederseits sind rostbräunlich; ähnlich, aber etwas heller, sind Brust und Leib gefärbt, der Hinterleib rostgelblich ohne scharfe Grenze. Kinn, Kehle und seitliche Halsflecken gelblichweiss. Auf dem Kopfe bewirkt das Durchscheinen der schwarzen Grundfärbung der einzelnen an der Spitze blauen Federn ein etwas gebändertes Aussehen. Die hellblauen Schaftstriche in dem blauen Theile der Kopf- und Flügeldeckfedern sind nicht so deutlich und daher das gestreifte Aussehen dieser Theile nicht so hervortretend wie bei lepida etc. - Von C. philippinensis und C. gentiana unterscheidet sich die Art leicht durch den vollständig korallenrothen Schnabel. Schwieriger ist vielleicht die Unterscheidung von $C$. sacerdotis Ramsay von Neu-Britannien, da diese Art bei verwandter Färbung des Gefieders nach Salvadori zu den rothschnäbeligen Formen gehören soll. - Ceyx Margarethae ist von Herrn Dr. Platen auch auf den Sulu-Inseln in einigen Exemplaren gesammelt worden, welche der Beschreibung mit zu Grunde gelegt sind.

Maasse, $\delta$ von Mindanao: Long. tot. $13 \mathrm{~cm}$. Ala $6,1 \mathrm{~cm}$. Cauda 2,1 cm. Culmen $3,4 \mathrm{~cm}$. Tarsus $0,95 \mathrm{~cm}$.

Typ.: đ̛ von Mindana0: Collection Nehrkorn; + von Sulu: Museum Brunsvicense.

Diesem Berichte füge ich noch hinzu, dass ein von Dr. Platen bei Davao gesammelter Vogelbalg, welcher vorläufig von mir der in den Listen der Mindanao-Vögel bereits angeführten Art Phylloscopus lugubris zugerechnet worden war, nachträglich als von der Originalbeschreibung und sicher bestimmten Exemplaren dieser Art abweichend erkannt wurde, so dass für diese Form vermuthlich eine besondere Benennung erforderlich werden wird.

Braunschweig, Herzogl. Naturhistorisches Museum, den 8. Mai 1890.

Wilh. Blasius. 


\section{$2 \mathrm{BHL}$ Biodiversity Heritage Library}

1890. "Die von Herrn Dr. Platen und dessen Gemahlin im Sommer 1889 bei Davao auf Mindanao gesammelten Vögel." Journal $f u$

r Ornithologie 38, 144-149. https://doi.org/10.1007/bf02250498.

View This Item Online: https://www.biodiversitylibrary.org/item/102239

DOI: https://doi.org/10.1007/bf02250498

Permalink: https://www.biodiversitylibrary.org/partpdf/142425

\section{Holding Institution}

Smithsonian Libraries

\section{Sponsored by}

Biodiversity Heritage Library

\section{Copyright \& Reuse}

Copyright Status: Public domain. The BHL considers that this work is no longer under copyright protection.

This document was created from content at the Biodiversity Heritage Library, the world's largest open access digital library for biodiversity literature and archives. Visit BHL at https://www.biodiversitylibrary.org. 